\title{
Constructing and Practicing Experiment Teaching of Electronic System Fault Diagnosis
}

\author{
Jiaoying Huang ${ }^{a}$, Chengcheng $\mathrm{Fu}^{\mathrm{b}}$ and Cheng Gao ${ }^{\mathrm{c}}$ \\ School of Reliability and System Engineering, Beihang University, Beijing 100191, China \\ ahuangjy@buaa.edu.cn, biamfcc@buaa.edu.cn, cgaocheng@buaa.edu.cn
}

\begin{abstract}
Firstly, an experiment teaching center, which in relation with electronic system fault diagnosis, was introduced in this paper, including the background, necessity and scheme of construction. Based on the fault diagnosis theory of analog and digital electronic system, two analog and digital electronic systems were improved, which is aimed at carrying out the student-oriented, combining theory with practice, and focusing on the students' practical ability and innovative spirit. After more than ten years of experimental teaching, good results were achieved. The construction of the experimental project can provide reference to relationship experimental teaching in domestic university.
\end{abstract}

Keywords: fault diagnosis; teaching system; experiment teaching; reliability; electronic system.

\section{Introduction}

With the rapid development of electronic technology, electronic products has penetrated into people's life. In recent years, the rise of large scale integrated circuit and the universal use of digital-analog mixed integrated circuit lead to more urgent demand of their test system.

No matter how high reliability a system or a product has, it can't work without fault forever. The user should monitor and test it to know its health condition, faults or where faults happen [1]. Due to the system has more and more big scale, high performance and complex structure, any failure of the components in the system may result in the failure of the whole system. So it is increasingly urgent requirement for the reliability, maintainability and the automatic fault diagnosis system of electronic circuits [2]. And people gradually realized that the reliability analysis and fault diagnosis of modern electronic system need to raise experience to the level of theory. At the same time, based on the solid theory, a set of exact methods of modern electric device fault analysis and diagnosis should be developed and improved. It should utilize the advanced technics of computer data process to realize the automatic detection, location, confirmation and fault prediction. To solve these problems is of significance for the reliability promotion of electric system for civil, aeronautic, astronautic and military use [3].

It is necessary and urgent to foster postgraduates and undergraduates' ability of modern electric device fault analysis, diagnosis and detection, improve their experimental skills and capability of comprehensive analyzation, problem-finding and problem-solving, which strengthens their innovation, entrepreneurship and practical ability [4].

The establishment of experimental teaching is pioneering for postgraduate experiment in domestic universities [5]. For domestic engineering graduates, this experimental teaching can enable them to master the technics of fault analysis and diagnosis of analog/digital circuit, have an intimate knowledge of testing instrument operation and further understanding of designing steps and compositions of automatic fault diagnosis system of electronic circuits. The experimental course will not only enable students get to know and master the rationale and latest development of fault diagnosis of analog/digital circuits, but also combine the modern electric device fault analysis and diagnosis and computer data processing, realizing the automatic detection, location, confirmation and fault prediction, which is beneficial for the graduates. 


\section{Electronic System Fault Diagnosis Experiment Teaching System}

Electronic system fault diagnosis experiment teaching system is constructed for the entire school, with the core of basic ability training, aiming at the cultivation of practice ability and innovation consciousness. According to different goals, set up different basic experiment, design experiment and comprehensive experiment [6]. Experiment teaching is constructed as an independent students oriented curriculum system to cultivate students' scientific experiment technology, scientific thinking and innovation ability, with multi-levels and multi-stages [7].

Electronic system fault diagnosis experiment is one of the experiment lessons in School of Reliability and System Engineering of Beihang University, as an innovation and practice platform for all postgraduate students. Based on postgraduate students develop system of school and characteristic of reliability engineering personnel, the experiment teaching equipment are designed, and the experiment guidebook is redacted [8]. The experiment teaching system is shown in Fig.1.

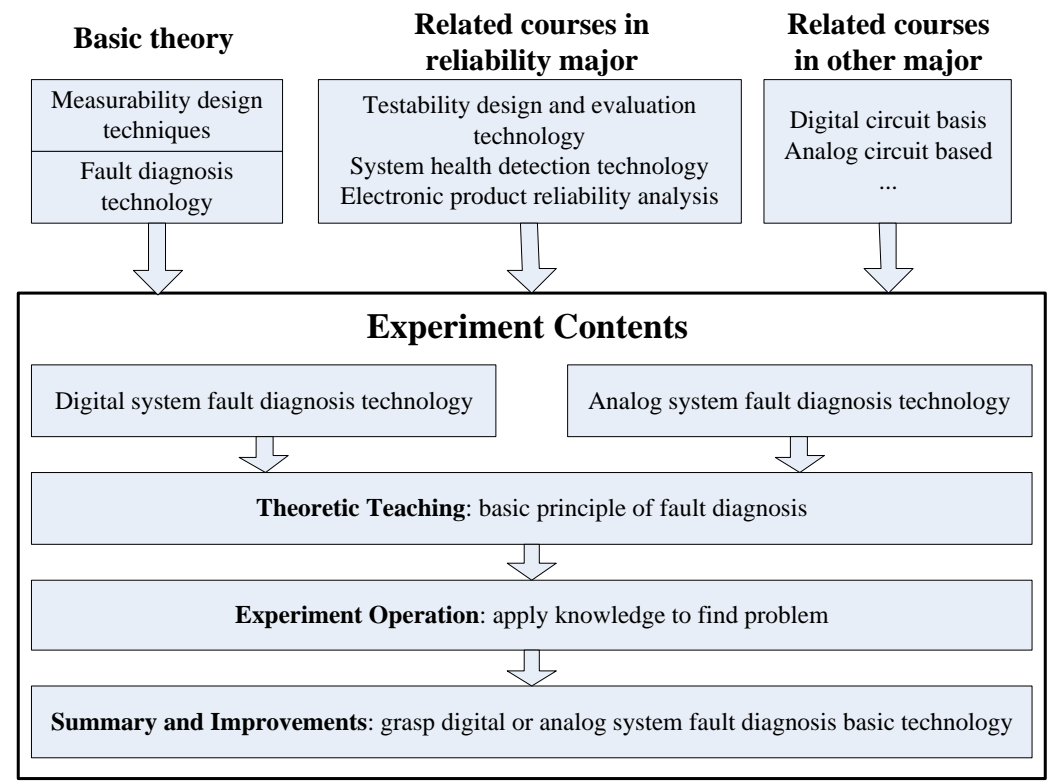

Fig.1 Experimental teaching system

\subsection{Experiment course construction content.}

The experiment course construction is centered on the basic methods of analog and digital circuit fault diagnosis and reliability design.

1) Fault diagnosis and reliability design of analog circuit

Learn and grasp Fault dictionary method for analog circuit fault diagnosis. Analyze and diagnose fault of typical analog circuit system.

2) Fault diagnosis and reliability design of digital circuit

Learn and grasp Pseudo-exhaustive testing method for combinational logic circuit fault testing and finding.

Learn and grasp method and principles of digital circuit fault dictionary construction. Use fault dictionary for fault testing and location.

Learn and grasp Boolean difference method to solve test pattern sets of fault and use testing tool in computer to locate fault and confirm fault mode.

\subsection{Experiment course content scope.}

Experiment course content covers major courses in components reliability field like Testability Design and Evaluation Technology and System Health Monitoring Technology and Electronic Product Reliability Analysis. It also can be appropriate as an experiment practice for basic courses, like Analog Circuit Based and Digital Circuit Based, of other majors.

Electronic system fault diagnosis experiment course is suit for postgraduate student and senior undergraduate students in the major of specialties of reliability, electronic, automation and computer related. 


\section{Delopment of Experiment Teaching Equipment}

As a fact that there is no readily available equipment for electronic system fault diagnosis experiment teaching, all the hardware and software experiment device are independently design and developed. It includes digital circuit system fault diagnosis experiment part and digital circuit system fault diagnosis experiment part. Every part includes corresponding hardware and software. Fig.2 to Fig.7 are the picture of hardware and software interface.

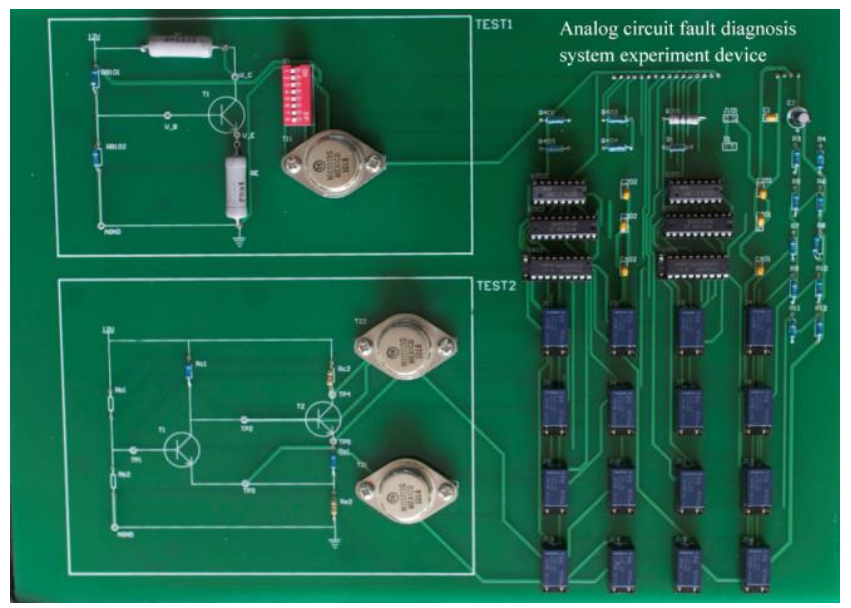

Fig. 2 Analog circuit fault diagnosis system experiment device.

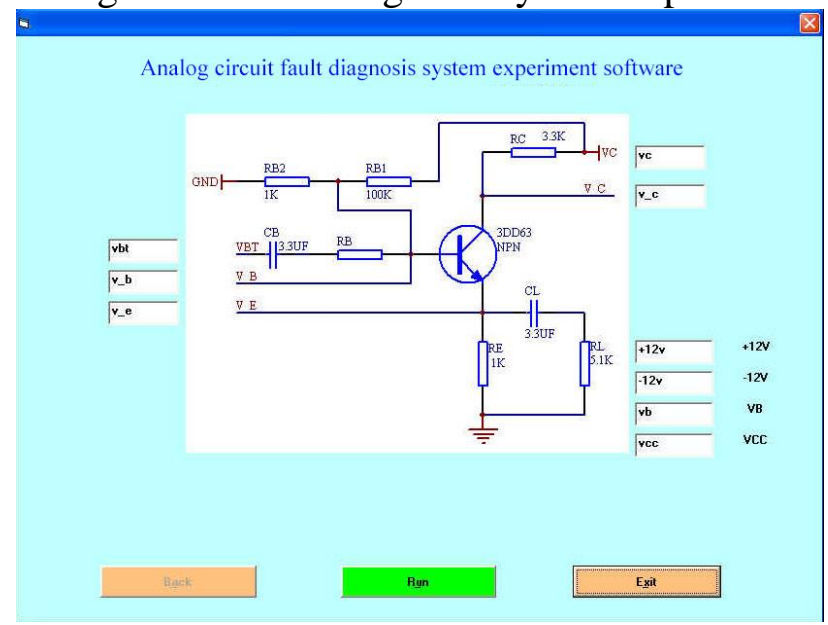

Fig.3 Analog circuit fault diagnosis system experiment software interface.

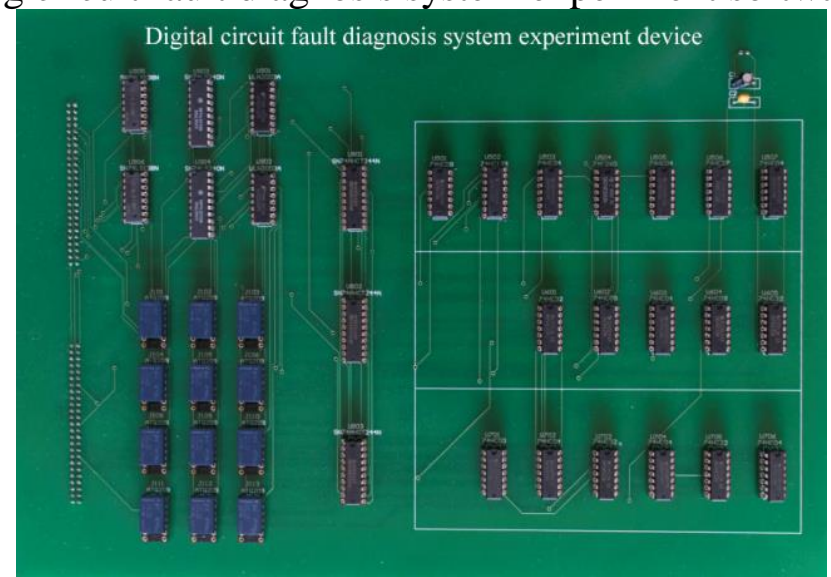

Fig.4 Digital circuit fault diagnosis system experiment device.

\section{Experiment Teaching Method and Means}

Theoretic teaching: teach basic principle and common method for analog and digital circuit fault diagnosis, 4 class hours.

Experiment operation: apply basic knowledge to solve practical problems, 4 class hours. 
Experiment report writing: 4 class hours.

Feedback, processing and generalizations: 2 class hours.

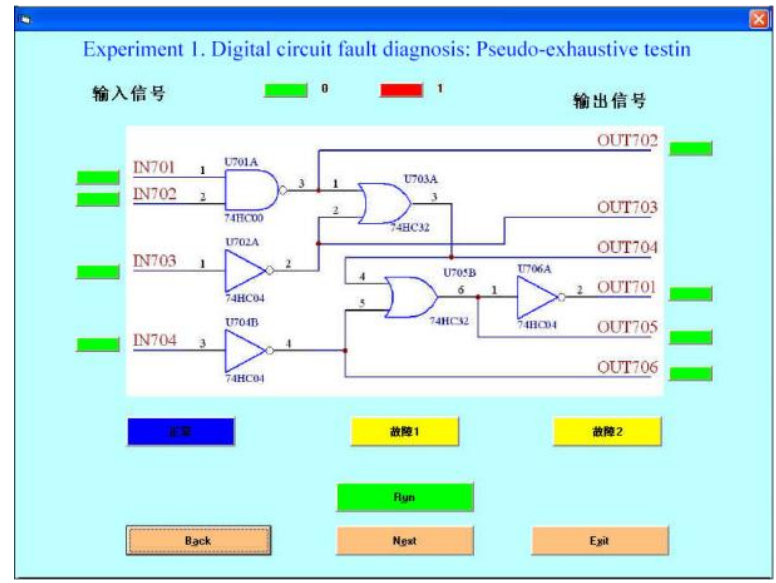

Fig.5 Digital circuit fault diagnosis system experiment software interface 1

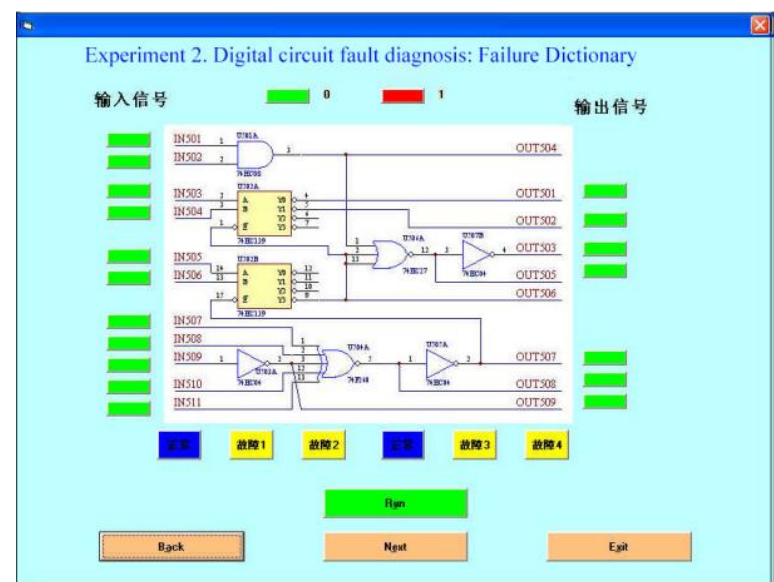

Fig.6 Digital circuit fault diagnosis system experiment software interface 2

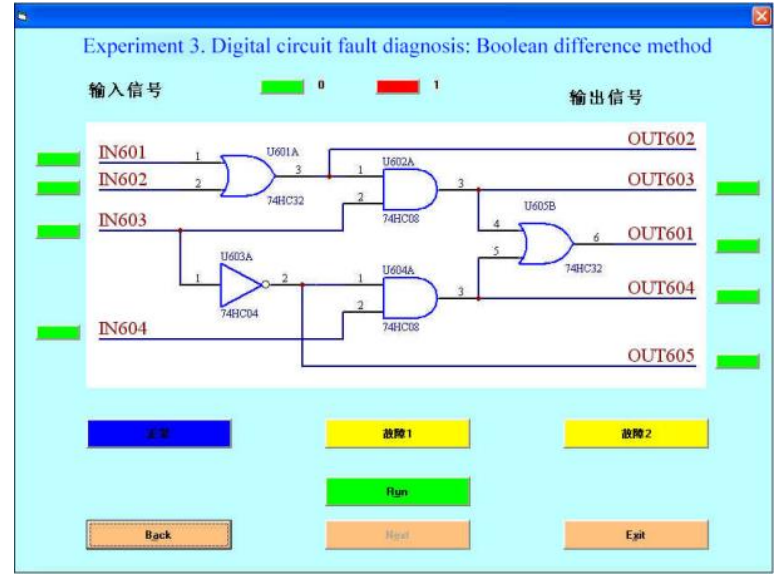

Fig.7 Digital circuit fault diagnosis system experiment software interface 3

\section{Operation mechanism}

All resource of experiment teaching is public online for students to practice open teaching. They can expediently get experimental framework, content, equipment and site for preview and review.

Experiment teaching itself is also opened for students. They can choose experiment time by appointment. Then students can enjoy autonomous operation under guidance of teacher. Free thinking 
is encouraged and teacher will provide corresponding condition for their verification. Question of the experiment principle and method are also encouraged to make experiment improved.

Experiment teaching need check and evaluation from expert panel composed of experienced experiment teachers every term. Expert panel member analyze the teaching situation by experiment class situation, experiment report and student feedbacks to find and solve problems and get experiment teaching well developed in the direction of more open and more perfect.

\section{Conclusion}

Since experiment teaching of electronic system fault diagnosis constructed, over 500 postgraduate students have attended the experiment class for over 7 thousands class hours, with good feedback from students.

The construction of electronic system fault diagnosis experiment teaching need the combination of theory teaching and experimental method, to improve operational and practical ability of students, to provide a stage for students. And experiment teacher need continuously explore, summarize and perfection.

\section{Acknowledgment}

This work was supported by technical basis research projects in Science and Industry Bureau of China under Grant No.Z132014A003, and Natural Science Foundation of China under Grant No.61201028 and 61376042.

\section{References}

[1]. ShiLi Ping, Lai Ping, \& Zhengting Gui. . "heat failure analysis of microwave power bipolar transistor," in Electronic Product Reliability and Environmental Testing. vol.4, pp. 16-18.(in Chinese)

[2]. GAO Cheng, HUANG Jiao-ying, SUN Yue and DIAO Sheng-long. Particle swarm optimization based RVM classifier for non-linear circuit fault diagnosis. Journal of Central South University, February 2012 ,vol.19, no.2, pp.459-564

[3]. Gao Cheng, Guo Wei, Sun, Yue. Research on diagnostics and prognostics of FPGA-based system. Proceedings of IEEE 2012 Prognostics and System Health Management Conference.

[4]. HUANG Jiun-lang, CHENG Wang-ting. Test point selection for analog fault diagnosis of unpowered circuit boards [J]. IEEE Transactions on Circuits and Systems-II: Analog and Digital Signal Processing, 2000, 47(10): 977-107.

[5]. BIPLAB K, SIKDAR, GANGULY N, CHAUDHERI P P. Fault diagnosis of VLSI circuits with cellular automata based pattern classifier [J]. IEEE Transaction Computer-Aided Design Integration Circuits System, 2005, 24(7): 1115-1131.

[6]. TAN Yang-hong, HE Yi-gang, CUI Chun. A novel method for analogfault diagnosis based on neural networks and genetic algorithms [J].IEEE Transactions on Instrumentation and Measurement, 2008,57(11): 2631-2639.

[7]. AMINIAN F, AMINIAN M. Neural-network based analog-circuit fault diagnosis using wavelet transform as preprocessor [J]. IEEE Transactions on Circuits and Systems-II: Analog and Digital Signal Processing, 2000, 47(2): 151-156.

[8]. YUAN Li-fen, HE Yi-gang, HUANG Jiao-ying, SUN Yi-chuang. A new neural network based fault diagnosis approach for analogcircuits by using kurtosis and entropy as a preprocessor [J]. IEEETransactions on Instruments and Measurement, 2010, 59(3): 586-595. 